\title{
EXISTENCIA Y UNICIDAD DE LA SOLUCIÓN GENERALIZADA DE UNA ECUACIÓN DIFERENCIAL PARABÓLICA QUE MODELA LA DISTRIBUCIÓN DE TEMPERATURA SOBRE LA FRONTERA DE UN DOMINIO CILÍNDRICO SOMETIDA A UNA FUERZA EXTERNA
}

\section{EXISTENCE AND UNITY OF THE GENERALIZED SOLUTION OF A PARABOLIC DIFFERENTIAL EQUATION THAT MODELS THE DISTRIBUTION OF TEMPERATURE ON THE BORDER OF A CYLINDRICAL DOMINION SUBJECT TO AN EXTERNAL STRENGTH}

\author{
Jhony Alfonso Chávez Delgado ${ }^{18}$, \\ Augusto Becerra Castañeda ${ }^{19}$, \\ Luis Alberto Chávez Delgado ${ }^{20}$, \\ Luis Asunción López Puycán ${ }^{21}$
}

\section{RESUMEN}

El propósito de este artículo matemático-físico es dar a conocer la aplicación de los espacios funcionales, es decir, el espacio de distribuciones, los espacio $L^{p}$ y el espacio de Sobolev en la teoría de la existencia y unicidad de la solución generalizada de una ecuación diferencial parabólica que modela la distribución de temperatura sobre la frontera de un dominio cilíndrico sometida a una fuerza externa. Este problema descrito por ecuaciones en derivadas parciales (EDP), pueden poseer como condiciones iniciales funciones (solución de dicha EDP) que no son regulares o suficientes para poseer funciones no diferenciable en el sentido clásico e incluso ser discontinua, he aquí la importancia de la solución débil o generalizada en el estudio de las ecuaciones de difusión. Se utilizó para el desarrollo del artículo el método deductivo para demostrar la existencia y unicidad de la solución generalizada del problema de evolución parabólico, qué consistió en aproximar la solución del problema por autofunciones del operador Laplaciano, y proyectando el espacio de Hilbert sobre una base de dimensión finita se construye la solución en un subespacio denso y separable. Luego lo dividimos en etapas: convergencia de las soluciones aproximadas en los espacios $\mathrm{L}^{2}\left(0, \mathrm{~T} ; \mathrm{L}^{2}(\Omega)\right)$ y $\mathrm{C}^{0}\left([0, \mathrm{~T}] ; \mathrm{H}_{0}^{1}(\Omega)\right)$, verificación de las condiciones iniciales y se demostró la unicidad de la solución generalizada.

Palabras claves: Ecuaciones diferenciales parabólicas, solución generalizada, existencia unicidad.

\footnotetext{
${ }^{18}$ Maestro en Ciencia con mención en Matemáticas. Docente de la Facultad de Ciencias de la Universidad Nacional Jorge Basadre Gronhmann. Tacna Perú

${ }^{19}$ Licenciado en Matemáticas. Docente de la Facultad de Ciencias de la Universidad Nacional Jorge Basadre Gronhmann. Tacna Perú.

${ }^{20}$ Licenciado en Educación. Docente del programa de formación general de la Universidad Particular Cesar Vallejo. Chimbote Perú.

${ }^{21}$ Magister en Docencia universitaria y Gestión educativa. Docente de la Facultad de Ciencias de la Universidad Nacional Jorge Basadre Gronhmann. Tacna Perú.
} 


\section{ABSTRACT}

parabolic differential that models the temperature distribution on the boundary of a cylindrical domain subjected to an external force. This problem described by partial differential equations

(PDE), can have as initial conditions functions (solution of said PDE) that are not regular or sufficient to have functions that can not be differentiated in the classical sense and even be discontinuous, here is the importance of the Weak or generalized solution in the study of diffusion equations. The deductive method was used for the development of the article to demonstrate the existence and uniqueness of the generalized solution of the problem of parabolic evolution, which consisted in approximating the solution of the problem by autofunctions of the Laplacian operator, and projecting the Hilbert space on a basis of finite dimension the solution is built in a dense and separable subspace. Then we divide it into stages: convergence of the approximate solutions in the spaces $\mathrm{L}^{2}\left(0, \mathrm{~T} ; \mathrm{L}^{2}(\Omega)\right)$ and $\mathrm{C}^{0}\left([0, \mathrm{~T}] ; \mathrm{H}_{0}^{1}(\Omega)\right)$, verification of the initial conditions and demonstrated the uniqueness of the generalized solution.

Keywords: Parabolic differential equations, generalized solution, existence of uniqueness.

\section{INTRODUCCIÓN}

La palabra clave del análisis es, desde luego, la de "función", y fue precisamente en la claridad de este término como fueron surgiendo la tendencia a la aritmetización. Durante la primera mitad del siglo XVIII habían aparecido diferencias de opinión sobre la manera de representar funciones, cuando D' Alembert y Euler dieron sus soluciones al problema de la cuerda vibrante, en la llamada "forma cerrada", utilizaron un par de funciones arbitrarias, mientras que Daniel Bernoulli había encontrado una solución en términos de una serie infinita de funciones trigonométricas .Así mismo, esta última solución parecía implicar claramente el carácter periódico de la función, mientras que las funciones arbitrarias de D' Alembert y de Euler no eran periódicas necesariamente, parecía que la solución de Bernoulli era menos general .Que no era éste el caso lo demostró al fin J.B.J Fourier en 1824(Boyer, 1986).

Los éxitos con el sonido y la gravitación animaron a los matemáticos a dirigir su atención hacia otros fenómenos físicos. Uno de los más importantes era el calor. A comienzos del siglo XIX la ciencia, del flujo de calor se estaba convirtiendo en un tema de gran interés práctico, principalmente a causa de las necesidades de la industria metalúrgica, pero también debido a un creciente interés en la estructura del interior de la Tierra, y en particular en la temperatura en el interior del planeta. No hay ninguna forma directa de medir la temperatura a mil kilómetros o más bajo la superficie de la Tierra, de modo que las únicas medidas disponibles eran indirectas y era esencial entender cómo fluía el calor a través de cuerpos de composiciones diferentes(Ian ,2012).

El primer paso de Fourier consistió en deriva una EDP para el flujo de calor. Con varias hipótesis simplificadoras: el cuerpo debe ser homogéneo (tiene las mismas propiedades en todas partes) e isótropo (se comporta de la misma manera en todas direcciones), y demás. Llegó a lo que ahora llamamos la ecuación del calor, que describe cómo cambia con el tiempo la temperatura en cualquier punto de un cuerpo tridimensional. La ecuación del calor tiene una forma muy similar a la ecuación de Laplace y la ecuación de la onda, pero la derivada parcial con respecto al tiempo es de primer orden, no de segundo. Este minúsculo cambio supone una profunda diferencia de las EDP (Lan ,2012).

Pero una ecuación diferencial parcial, por si sola, no es suficiente para dar información 
concreta sobre un determinado problema. Al estudiar a las EDP es necesario dar condiciones extras, que fundamentalmente, son del tipo: condiciones iniciales y/o condiciones de contorno. Así en forma natural surgieron los dos problemas básicos de la teoría: el problema de Cauchy y el problema de valor en la frontera. El estudio del problema de Cauchy y del problema del valor en la frontera dieron las motivaciones hacia un análisis más generalizado. Trabajos pioneros son debidos, entre otros, a Sobolev (1935-1936), Oseen (1911), Leray (1934), entre otros. Por la línea de los espacios de Hilbert tenemos los aportes de Friedrichs, Calkin, Sobolev, Neumann. (Ortiz, 1988).

La aparición de la "función "delta de Dirac, conocida desde la época de Fourier, jugo un papel central en el manejo de nuevas ideas, lo que se fortaleció con uso por parte de Heaviside, y sobre el trabajo de Dirac en el estudio de la mecánica cuántica. Todo ello, entre otros aspectos, fue consolidado con nuevos aportes, por el matemático francés Laurent Schwartz al idealizar la teoría de las distribuciones (1940); se abre así una nueva era en el estudio de las ecuaciones en derivadas parciales (Ortiz, 1988).

Consideremos una ecuación parabólica, representado por un modelo matemático referente a que modela la distribución de temperatura sobre la frontera de un dominio cilíndrico sometida a una fuerza externa. Para una ecuación de este tipo, las condiciones de Dirichlet expresa que la barra elástica esta fija en el borde de la frontera y el estado inicial del sistema son los datos de Cauchy: la configuración inicial (también llamado desplazamiento inicial) y la velocidad inicial (Brezis, 1984).

Desde el punto de vista físico este modelo parabólico requiere que la existencia de su solución sea físicamente aceptable y "controlable". Matemáticamente esto se traduce en un problema de existencia, unicidad y dependencia continua de las condiciones iniciales y $/ 0$ condiciones de contorno (Vera, 1984).
Pero, una dificultad presenta la ecuación de evolución parabólica, como por ejemplo, no se tiene una solución generalizada para la existencia y unicidad. Así, frente a la dificultad, el análisis funcional es una herramienta fundamental en la investigación de los problemas de evolución, más precisamente, espacios funcionales dentro de la teoría de las distribuciones son usadas para el estudio de los teoremas de existencia y unicidad de la solución generalizada de las ecuaciones en derivadas parciales, las cuales han sido exitosamente usados por muchos matemáticos de gran prestigio como Adams (1975); Brézis (1984); Medeiros (1990).

\section{MARCO TEÓRICO}

\section{Teoría de Distribuciones}

Los espacios serán definidos sobre un conjunto abierto $\Omega \subset \mathbb{R}^{n}$.

Definición (Soporte de una función) Sea $\varphi$ una función real de variable vectorial. Se denomina soporte de $\varphi$ al conjunto $\operatorname{sop} \varphi=\overline{\left\{\mathrm{x} \in \mathbb{R}^{\mathrm{n}} / \varphi(\mathrm{x}) \neq 0\right\}}$.

Definición (EspacioC $C_{0}^{\infty}(\Omega)$ ). El espacio $\mathrm{C}_{0}^{\infty}(\Omega)$ es el espacio vectorial de las funciones infinitamente diferenciables en $\Omega$ $y$ tienen su soporte contenido en un compacto de $\Omega$.

Definición (Convergencia) Una sucesión de funciones $\left(\varphi_{\mathrm{j}}\right)$ converge para para $\varphi$ en $\mathrm{C}_{0}^{\infty}(\Omega)$, si satisface las siguientes condiciones:

a) $\operatorname{sop}\left(\varphi_{\mathrm{j}}\right) \subset \mathrm{K}$, donde $\mathrm{K}$ es un compacto fijo en $\Omega, \forall j=1,2, \ldots$

b) $\left(\varphi_{\mathrm{j}}\right)$ converge uniformemente para $\varphi$ en $\Omega$, juntamente con todas sus derivadas de cualquier orden.

Definición (Espacio de las funciones de prueba) El espacio de las funciones de prueba es el espacio vectorial $\mathrm{C}_{0}^{\infty}(\Omega)$ con la convergencia en $\mathrm{C}_{0}^{\infty}(\Omega)$

Definición

(Espacio de Distribución). Una distribución sobre $\Omega$ es una funcional lineal $T$, continua en el espacio de las funciones de prueba $\mathrm{D}(\Omega)$. 
Definición (Derivada de una distribución) La derivada de una distribución T sobre un abierto $\Omega$ de $\mathbb{R}^{\mathrm{n}}$ es una funcional lineal $\frac{\partial T}{\partial x_{k}}$, continua en el espacio de las funciones de prueba $D(\Omega)$, definida por la relación

$$
\left\langle\frac{\partial \mathrm{T}}{\partial \mathrm{x}_{\mathrm{k}}}, \varphi\right\rangle=-\left\langle\mathrm{T}, \frac{\partial \varphi}{\partial \mathrm{x}_{\mathrm{k}}}\right\rangle, \forall \varphi \in \mathrm{D}(\Omega), \mathrm{k}=\overline{1, \mathrm{n} .}
$$

\section{Espacios de Sobolev}

Sea $\Omega \subset \mathbb{R}^{n}$ un abierto y sea $\mathrm{p} \in \mathbb{R}$ con $1 \leq$ $\mathrm{p} \leq \infty$.

Definición (El espacio $\mathrm{W}^{\mathbf{1}, \mathrm{p}}(\Omega)$ ) El espacio de Sobolev $\mathrm{W}^{1, \mathrm{p}}(\Omega)$ es el conjunto de funciones $u \in L^{p}(\Omega)$, tales que todas las derivadas parciales $\frac{\partial u}{\partial x_{i}}, i=1,2, \ldots, n$, pertenecen a $\mathrm{L}^{\mathrm{p}}(\Omega)$, en el sentido de las distribuciones sobre $\Omega$.

El espacio $\mathrm{W}^{1, \mathrm{p}}(\Omega)$ está dotado de la norma

$$
\|\mathrm{u}\|_{\mathrm{w}^{1, p}}^{\mathrm{p}}=\|\mathrm{u}\|_{\mathrm{L}^{\mathrm{p}}}^{\mathrm{p}}+\sum_{\mathrm{i}=1}^{\mathrm{n}}\left|\frac{\partial \mathrm{u}}{\partial \mathrm{x}_{\mathrm{i}}}\right|_{\mathrm{L}^{\mathrm{p}}}^{\mathrm{p}}
$$

El espacio $\mathrm{W}^{1, \mathrm{p}}(\Omega)$ es un espacio de Banach para $1 \leq p \leq \infty ; \mathrm{W}^{1, \mathrm{p}}(\Omega)$ es reflexivo para $1<p<\infty$ y separable para $1 \leq p<\infty$.

Notación $\mathrm{H}^{1}(\Omega)=\mathrm{W}^{1, \mathrm{p}}(\Omega)$, es el espacio de Sobolev de orden uno.

(Adams, 1975)

\section{Definición (El espacio $\mathrm{W}_{0}^{1, p}(\Omega)$ )}

Sea $p \in \mathbb{R}$ con $1 \leq \mathrm{p}<\infty$, se define el espacio $\mathrm{W}_{0}^{1, \mathrm{p}}(\Omega)$ como la cerradura de $\mathrm{D}(\Omega)$ en $\mathrm{W}^{1, \mathrm{p}}(\Omega)$, el cual está dotado de la norma $\quad\|\mathrm{u}\|_{\mathrm{w}_{0}^{1, \mathrm{p}}}^{\mathrm{p}}=\sum_{\mathrm{i}=1}^{\mathrm{n}}\left|\frac{\partial \mathrm{u}}{\partial \mathrm{x}_{\mathrm{i}}}\right|_{\mathrm{L}^{\mathrm{p}}}^{\mathrm{p}}$

El espacio $\mathrm{W}_{0}^{1, \mathrm{p}}(\Omega)$ dotado de la norma inducida por $\mathrm{W}^{1, \mathrm{p}}(\Omega)$ es un espacio de Banach separable; es reflexivo si $1 \leq p<\infty$ (Adams, 1975)

(Definición (EI espacioH $\mathbf{H}_{0}^{1}(\Omega)$ ) Se define el espacio $\mathrm{H}_{0}^{1}(\Omega)$ como la cerradura de $\mathrm{D}(\Omega)$ en $\mathrm{H}^{1}(\Omega)$, el cual está dotado con la norma

$$
\|\mathrm{u}\|_{\mathrm{H}_{0}^{1}}^{2}=\sum_{\mathrm{i}=1}^{\mathrm{n}}\left|\frac{\partial \mathrm{u}}{\partial \mathrm{x}_{\mathrm{i}}}\right|_{\mathrm{L}^{2}}^{2}
$$

El espacio $\mathrm{H}_{0}^{1}(\Omega)$ es un espacio de Hilbert separable, con el producto escalar de $\mathrm{H}^{1}(\Omega)$.

(Adams, 1975)

\section{Definición (El espacio $\mathrm{L}^{\mathrm{P}}(\boldsymbol{\Omega})$ )}

Sea $\Omega$ un abierto de $\mathbb{R}^{\mathrm{n}}$.Denotaremos por $\mathbf{L}^{\mathbf{P}}(\boldsymbol{\Omega}), \mathbf{1} \leq \boldsymbol{p}<\infty$, el espacio vectorial de funciones $\varphi$ definidas en $\boldsymbol{\Omega}$ con valores en $\mathbb{K}$, medible, tal que $|\varphi|^{p}$ es integrable en el sentido de Lebesgue.

(Medeiros, 1990)

Propiedades que serán usados para la existen y unicidad de la solución generalizada

Lema de Gronwall Sea $\varphi:(0, T) \rightarrow \mathbb{R}$ absolutamente continúa tal que

$\varphi(\mathrm{t}) \geq 0 \forall \mathrm{t} \in(0, \mathrm{~T}) . \mathrm{Si}$

$\varphi(\mathrm{t}) \leq \mathrm{c}_{1}+\mathrm{c}_{2} \int_{0}^{\mathrm{t}} \varphi(\mathrm{s}) \mathrm{ds}$, entonces $\varphi(\mathrm{t}) \leq \mathrm{c}$ (Medeiros, 1990)

Desigualdad de Holder Sean $p \in[1, \infty>$ y $q \in[1, \infty>$ su exponente conjugado, entonces

$\int_{\Omega}|u v| \leq\|u\|_{p ; \Omega}\|v\|_{q ; \Omega} ; \forall u \in L^{p}(\Omega), v$

$$
\in L^{q}(\Omega)
$$

Para $p$ y $q$ en $\langle 1, \infty\rangle$ la desigualdad del Holder se escribe

$\int_{\Omega}|u v| \leq\left(\int_{\Omega}|u|^{p}\right)^{1 / p}\left(\int_{\Omega}|u|^{q}\right)^{1 / q} ; \forall u$ $\in L^{p}(\Omega), v \in L^{q}(\Omega)$

En particular si $p=q=2$ se tiene la desigualdad de Schwartz.

$$
\begin{gathered}
\int_{\Omega}|u v| \leq\left(\int_{\Omega}|u|^{2}\right)^{1 / 2}\left(\int_{\Omega}|u|^{2}\right)^{1 / 2} ; \forall u \\
\in L^{2}(\Omega), v \in L^{2}(\Omega)
\end{gathered}
$$

(Brézis, 1984)

Desigualdad de Minkowsky $\left(\int_{\Omega}|u+v|^{p}\right)^{1 / p}$
(Brézis, 1984)

$$
\begin{aligned}
& \leq\left(\int_{\Omega}|u|^{p}\right)^{1 / p} \\
& +\left(\int_{\Omega}|u|^{q}\right)^{1 / q} ; \forall u \in L^{p}(\Omega), v \\
& \in L^{q}(\Omega)
\end{aligned}
$$


En éste artículo es considerado material de estudio un modelo matemático referente a la distribución de temperatura (propagación del calor, etc.) en un dominio $\Omega \subset \mathbb{R}^{n}$ en el instante t.

Sea $\Omega$ un conjunto abierto, acotado y bien regular de $\mathbb{R}^{n}$, cuya frontera lo denotamos por $\Gamma$. Así mismo, se denota $\mathrm{Q}=\Omega \times] 0, \infty[$,

$\Sigma=\Gamma \mathrm{x}] 0, \infty[; \Sigma$ es la frontera lateral del cilindro $Q$.

Consideremos el problema siguiente en que dados $\mathrm{u}_{0} \in \mathrm{L}^{2}(\Omega)$ y $\mathrm{f} \in \mathrm{L}^{2}(\mathrm{Q})$ hallar una función real $u=u(x, t)$ definida en $Q$, tal que

$$
\left\{\begin{array}{c}
\frac{\partial \mathrm{u}}{\partial \mathrm{t}}-\Delta \mathrm{u}=\mathrm{f} \text { en } \mathrm{Q} \\
\mathrm{u}=0 \quad \text { sobre } \Sigma \\
\mathrm{u}(\mathrm{x}, 0)=\mathrm{u}_{0}(\mathrm{x}) \text { en } \Omega
\end{array}\right.
$$

, donde $\Delta=\sum_{i=1}^{n} \frac{\partial^{2}}{\partial x_{i}^{2}}$ designa el Laplaciano respecto de las variables espaciales, $t$ es la variable tiempo, $\mathrm{u}_{0} \mathrm{y} \mathrm{u}_{1}$ son funciones dadas (Medeiros ,1990).

El método usado es el método de Faedo Galerkin-Medeiro que consiste en obtener una secuencia aproximada del problema (1.1), por medio de estimativa a priori la sucesión que converge para la solución del problema.

\section{RESULTADOS}

\section{Existencia de la solución generalizada} de la distribución de temperatura:

Existe una solución débil o generalizada para el problema parabólico (1.1), es decir una función

$\mathrm{u} \in \mathrm{C}^{0}\left([0, \mathrm{~T}] ; \mathrm{H}_{0}^{1}(\Omega)\right) \cap \mathrm{L}^{2}\left(0, \mathrm{~T} ; \mathrm{L}^{2}(\Omega)\right)$ satisfaciendo:

$\frac{\mathrm{d}}{\mathrm{dt}}\left(\mathrm{u}^{\prime}(\mathrm{t}) ; \mathrm{v}\right)+\mathrm{a}(\mathrm{u}(\mathrm{t}) ; \mathrm{v})=(\mathrm{f}(\mathrm{t}) ; \mathrm{v}) \forall \mathrm{v} \in$ $\mathrm{H}_{0}^{1}(\Omega)$, en el sentido de $\mathrm{D}^{\prime}(0 ; \mathrm{T})$.

Unicidad de la solución generalizada de la distribución de temperatura:

La solución generalizada $\mathrm{u} \in$ $\mathrm{C}^{0}\left([0, \mathrm{~T}] ; \mathrm{H}_{0}^{1}(\Omega)\right) \cap \mathrm{L}^{2}\left(0, \mathrm{~T} ; \mathrm{L}^{2}(\Omega)\right)$ del problema parabólico (1.1) es única.

\section{DISCUSIÓN}

La matemática, en tanto es una ciencia eidética o formal - como dice Bunge utiliza el método deductivo, es decir, que mediante deducciones lógicas, obtiene verdades $\mathrm{o}$ afirmaciones a partir de axiomas previamente formuladas.
Existencia y unicidad de la solución generalizada de la distribución de temperatura

Teorema. Sean $f \in L^{2}\left(0, T ; L^{2}(\Omega)\right)$,

$\mathrm{u}_{0} \in \mathrm{H}_{0}^{1}(\Omega)$. Entonces existe una única función

$\mathrm{u} \in \mathrm{C}^{0}\left([0, \mathrm{~T}] ; \mathrm{H}_{0}^{1}(\Omega)\right) \cap$ $\mathrm{L}^{2}\left(0, \mathrm{~T} ; \mathrm{L}^{2}(\Omega)\right)$

Solución generalizada de (1.1), que satisface:

$$
\frac{d}{d t}(u(t), v)+a(u(t), v)=(f(t), v)
$$

$\forall \mathrm{v} \in \mathrm{H}_{0}^{1}(\Omega)$ en el sentido de $\mathrm{D}^{\prime}(0, \mathrm{~T})$

\section{Demostración}

$$
u(0)=u_{0}
$$

Considere los autovalores del Laplaciano $-\Delta: \lambda_{1}<\lambda_{2}<\lambda_{3}<\cdots<\lambda_{v}<$ $\cdots \mathrm{y}$, sus autofunciones $\mathrm{w}_{1}, \mathrm{w}_{2}, \ldots, \mathrm{w}_{v}, \ldots$ la cual es una base ortonormal completo en $\mathrm{H}_{0}^{1}(\Omega)$ tal que

$$
\mathrm{a}\left(\mathrm{w}_{\mathrm{v}}, \mathrm{v}\right)=\lambda_{v}\left(\left(\mathrm{w}_{v}, \mathrm{v}\right)\right), \forall \mathrm{v} \in \mathrm{H}_{0}^{1}(\Omega)
$$

Sea el subespacio

$$
\mathrm{V}_{\mathrm{m}}=\overline{\left[\mathrm{w}_{1}, \mathrm{w}_{2}, \ldots, \mathrm{w}_{\mathrm{m}}\right]}=\mathrm{H}_{0}^{1}(\Omega)
$$

, y considere la solución aproximada $\mathrm{u}_{\mathrm{m}}:\left[0, \mathrm{t}_{\mathrm{m}}\right] \rightarrow \mathrm{H}_{0}^{1}(\Omega)$, definida como la serie

$\mathrm{u}_{\mathrm{m}}(\mathrm{t})=\sum_{v=1}^{\mathrm{m}}\left(\mathrm{u}_{\mathrm{m}}(\mathrm{t}), \mathrm{w}_{v}\right) \mathrm{w}_{v} \quad$, que es solución del problema aproximado:

$\left\{\begin{array}{l}\frac{d}{d t}\left(u_{m}(t), v\right)+a\left(u_{m}(t), v=(f(t), v) \quad \forall v \in V_{m} \text { en } D^{\prime}(0, T)\right) \\ u_{m}(0)=u_{0 m}, u_{0 m}=\sum_{v=1}^{m}\left(u_{m}(t), w_{v}\right) w_{v} \rightarrow u_{0} \text { en } H_{0}^{1}(\Omega)\end{array}\right.$

La demostración del Teorema se divide en etapas:

Etapa1. Convergencia de las soluciones aproximadas en el espacio

$\mathbf{L}^{2}\left(\mathbf{0}, \mathbf{T} ; L^{2}(\Omega)\right)$ y $\mathbf{C}^{0}\left([\mathbf{0}, \mathbf{T}] ; \mathbf{H}_{0}^{1}(\Omega)\right)$ :

Sea $v=w_{v}$ en (2), se tiene que

$\frac{\mathrm{d}}{\mathrm{dt}}\left(\left(\mathrm{u}_{\mathrm{m}}(\mathrm{t}), w_{v}\right)\right)+\lambda_{v}\left(\left(\mathrm{u}_{\mathrm{m}}(\mathrm{t}), w_{v}\right)\right)=$

$\left(\mathrm{f}(\mathrm{t}), w_{v}\right) \quad$ (3) $\forall w_{v} \in \mathrm{V}_{\mathrm{m}}$, en el sentido de la $\mathrm{D}^{\prime}(0, \mathrm{~T})$.

La ecuación (3) tiene la forma de una E.D.O:

$$
\begin{gathered}
\frac{\mathrm{d}}{\mathrm{dt}} \mathrm{y}(\mathrm{t})+\lambda_{v} \mathrm{y}(\mathrm{t})=\mathrm{g}(\mathrm{t}) \Leftrightarrow\left(\mathrm{ye}^{\lambda_{v} \mathrm{t}}\right)^{\prime} \\
=\mathrm{e}^{\lambda_{v} \mathrm{t}} \mathrm{g}(\mathrm{t}) \\
\Leftrightarrow y(t) e^{\lambda_{v} t}-y(0)=\int_{0}^{t} e^{\lambda_{v} s} g(s) d s \\
\Leftrightarrow y(t)=y_{0} e^{-\lambda_{v} t}+\int_{0}^{t} e^{\lambda_{v}(s-t)} g(s) d s
\end{gathered}
$$

, y entonces 
$\left(\left(\mathrm{u}_{\mathrm{m}}(\mathrm{t}), w_{v}\right)\right)$

$=\left(\left(\mathrm{u}_{0 \mathrm{~m}}(\mathrm{t}), w_{v}\right)\right) e^{-\lambda_{v} t}$

$+\int_{0}^{t} e^{-\lambda_{v}(t-s)}\left(\mathrm{f}(\mathrm{t}), w_{v}\right) d s$

Por la Identidad de Parseval se tiene $\left|u_{m}(t)-u_{n}(t)\right|_{L^{2}(\Omega)}^{2}=\sum_{v=n}^{m}\left[\left(\left(\mathrm{u}_{\mathrm{m}}(\mathrm{t}), \mathrm{v}\right)\right)\right]^{2}$

(5)

Reemplazando (4) en (5) se obtiene $\left|u_{m}(t)-u_{n}(t)\right|_{L^{2}(\Omega)}^{2}=$

$\sum_{v=n}^{m}\left[\left(\left(\mathrm{u}_{0 \mathrm{~m}}(\mathrm{t}), w_{v}\right)\right) e^{-\lambda_{v} t}+\right.$

$$
\left.+\int_{0}^{t} e^{-\lambda_{v}(t-s)}\left(\mathrm{f}(\mathrm{t}), w_{v}\right) d s\right]^{2}
$$

Aplicando la desigualdad de Minkowsky en (6) se tiene

$$
\begin{aligned}
& \left|u_{m}(t)-u_{n}(t)\right|_{L^{2}(\Omega)}^{2} \\
& \leq\left(\sum_{v=n}^{m}\left|\left(\left(\mathrm{u}_{0 \mathrm{~m}}(\mathrm{t}), w_{v}\right)\right) e^{-\lambda_{v} t}\right|^{2}\right)^{\frac{1}{2}} \\
& +\left(\sum_{v=n}^{m}\left|e^{-\lambda_{v}(t-s)}\left(\mathrm{f}(\mathrm{t}), w_{v}\right) d s\right|^{2}\right)^{1 / 2} \\
& \left(\sum_{v=n}^{m}\left|\left(\left(\mathrm{u}_{0 \mathrm{~m}}(\mathrm{t}), w_{v}\right)\right) e^{-\lambda_{v} t}\right|^{2}\right)^{1 / 2}+ \\
& +\left(\sum_{v=n}^{m}\left|\int_{0}^{t} e^{-\lambda_{v}(t-s)}\left(\mathrm{f}(\mathrm{t}), w_{v}\right) d s\right|^{2}\right)^{1 / 2} \\
& \text { y por tanto se obtiene } \\
& )\left|u_{m}(t)-u_{n}(t)\right|_{L^{2}(\Omega)}^{2} \leq \\
& \left(\sum_{v=n}^{m}\left|\left(\left(\mathrm{u}_{0 \mathrm{~m}}(\mathrm{t}), w_{v}\right)\right)\right|^{2}\right)^{\frac{1}{2}}+ \\
& +\left(\sum_{v=n}^{m}\left|\int_{0}^{t} e^{-\lambda_{v}(t-s)}\right|^{2} d s \int_{0}^{t}\left|\left(\mathrm{f}(\mathrm{t}), w_{v}\right)\right|^{2} d s\right)^{\frac{1}{2}}
\end{aligned}
$$

Pero, de (7) se tiene que

$$
\begin{aligned}
\left|\int_{0}^{t} e^{-\lambda_{v}(t-s) 2}\right|= & \left|\frac{e^{-\lambda_{v} 2 t}}{2 \lambda_{v}}\left(e^{\lambda_{v} t}-1\right)\right|= \\
& =\left|\frac{1}{2 \lambda_{v}}\left(e^{-\lambda_{v} t}-e^{-\lambda_{v} 2 t}\right)\right| \leq
\end{aligned}
$$

$\frac{1}{2 \lambda_{1}} 2=\frac{1}{\lambda_{1}}$.

De (7) y de (8) se obtiene que

$\left(\sum_{v=n}^{m}\left|\int_{0}^{t} e^{-\lambda_{v}(t-s)}\right|^{2} d s \int_{0}^{t}\left|\left(\mathrm{f}(\mathrm{t}), w_{v}\right)\right|^{2} d s\right)^{\frac{1}{2}}=$ $\left(\frac{1}{\lambda_{1}} \int_{0}^{t} \sum_{v=n}^{m}\left|\left(\mathrm{f}(\mathrm{t}), w_{v}\right)\right|^{2} d s\right)^{1 / 2} \rightarrow 0$ si $\mathrm{m}, \mathrm{n} \rightarrow$ $\infty$

Luego, $\mathrm{f} \in \mathrm{L}^{2}\left(0, \mathrm{~T} ; \mathrm{L}^{2}(\Omega)\right)$, y $\|\mathrm{f}\|_{\mathrm{L}^{2}(\Omega)} \leq \mathrm{c}$ donde $\mathrm{f}(\mathrm{t})=\sum_{v=1}^{\mathrm{m}}\left(\mathrm{f}(\mathrm{t}), \mathrm{w}_{v}\right) \mathrm{w}_{v}$.Por tanto $\left|u_{m}(t)-u_{n}(t)\right|_{L^{2}(\Omega)}^{2} \rightarrow 0 \quad$, entonces $\left(u_{m}\right)$ es de cauchy en $L^{2}(\Omega)$.

De igual manera tenemos

$$
\begin{aligned}
\left|u_{m}-u_{n}\right|_{C^{0}\left([0, T], \mathrm{L}^{2}(\Omega)\right)} & \\
& =\max _{0 \leq t \leq T}\left|u_{m}(t)-u_{n}(t)\right|_{L^{2}(\Omega)} \\
& \rightarrow 0
\end{aligned}
$$

$y$ se concluye que

Si $\left(u_{m}\right)$ es de cauchy en $C^{0}\left([0, T], L^{2}(\Omega)\right)$

entonces $u_{m} \rightarrow \mathrm{u}$ en $\mathrm{C}^{0}\left([0, \mathrm{~T}], \mathrm{L}^{2}(\Omega)\right)$

Si $\left(\mathrm{u}_{\mathrm{m}}\right)$ es de cauchy en $\mathrm{C}^{0}\left([0, \mathrm{~T}], \mathrm{H}_{0}^{1}(\Omega)\right)$ entonces $u_{m} \rightarrow$ u en $\mathrm{C}^{0}\left([0, \mathrm{~T}], \mathrm{H}_{0}^{1}(\Omega)\right)$.

$\mathrm{De} \quad(2)$ tenemos que

$\frac{d}{d t}\left(u_{m}(t), v\right)+a\left(u_{m}(t), v\right)=(f(t), v)$

(9)

$\forall \mathrm{v} \in \mathrm{V}_{\mathrm{m}}$ en el sentido $\mathrm{D}^{\prime}(0, \mathrm{~T})$

y multiplicando por $\theta \in \mathrm{D}(0, \mathrm{~T})$ e

integrando de 0 a $T$ en (9) se obtiene

$-\int_{0}^{\mathrm{T}}\left(u_{m}(t), \mathrm{v}\right) \frac{d}{d t} \theta(\mathrm{t}) \mathrm{dt}+$

$\int_{0}^{\mathrm{T}} \mathrm{a}\left(u_{m}(\mathrm{t}), \mathrm{v}\right) \theta(\mathrm{t}) \mathrm{dt}=$

$\int_{0}^{\mathrm{T}}(\mathrm{f}(\mathrm{t}), \mathrm{v}) \theta(\mathrm{t}) \mathrm{dt} \forall \theta \in \mathrm{D}(0, \mathrm{~T})$

(10)

Aplicando la convergencia a las expresiones en (10) se obtiene

$\int_{0}^{\mathrm{T}}(u(t), \mathrm{v}) \frac{d}{d t} \theta(\mathrm{t}) \mathrm{dt}+\int_{0}^{\mathrm{T}} \mathrm{a}(\mathrm{u}(\mathrm{t}), \mathrm{v}) \theta(\mathrm{t}) \mathrm{dt}=$ $\int_{0}^{\mathrm{T}}(\mathrm{f}(\mathrm{t}), \mathrm{v}) \theta(\mathrm{t}) \mathrm{dt} \forall \theta \in \mathrm{D}(0, \mathrm{~T})$.

(11)

De (11) se obtiene

$$
\begin{gathered}
\int_{0}^{\mathrm{T}} \frac{d}{d t}(u(t), \mathrm{v}) \theta(\mathrm{t}) \mathrm{dt}+\int_{0}^{\mathrm{T}} \mathrm{a}(\mathrm{u}(\mathrm{t}), \mathrm{v}) \theta(\mathrm{t}) \mathrm{dt} \\
=\int_{0}^{\mathrm{T}}(\mathrm{f}(\mathrm{t}), \mathrm{v}) \theta(\mathrm{t}) \mathrm{dt} \quad \forall \theta \in \mathrm{D}(0, \mathrm{~T})
\end{gathered}
$$

, y resulta

$$
\frac{\mathrm{d}}{\mathrm{dt}}(\mathrm{u}(\mathrm{t}), \mathrm{v})+\mathrm{a}(\mathrm{u}(\mathrm{t}), \mathrm{v})=(\mathrm{f}(\mathrm{t}), \mathrm{v})
$$

$\forall \mathrm{v} \in \mathrm{V}_{\mathrm{m}}$ en sentido $\mathrm{D}^{\prime}(0, \mathrm{~T})$.

Por tanto $\overline{V_{m}}=\mathrm{H}_{0}^{1}(\Omega), \mathrm{y}$

$$
\begin{gathered}
\frac{\mathrm{d}}{\mathrm{dt}}(\mathrm{u}(\mathrm{t}), \mathrm{v})+\mathrm{a}(\mathrm{u}(\mathrm{t}), \mathrm{v})=(\mathrm{f}(\mathrm{t}), \mathrm{v}) \forall \mathrm{v} \\
\in \mathrm{H}_{0}^{1}(\Omega)
\end{gathered}
$$

Etapa2.Verificación de las condiciones iniciales:

Se sabe que $\mathrm{u}_{\mathrm{m}} \rightarrow \mathrm{u}$ en $\mathrm{C}^{0}\left([0, \mathrm{~T}], \mathrm{L}^{2}(\Omega)\right)$

En particular en el origen, se tiene

$$
\begin{aligned}
& \mathrm{u}_{\mathrm{m}}(0) \rightarrow \mathrm{u}(0) \text { en } \mathrm{C}^{0}\left([0, \mathrm{~T}], \mathrm{L}^{2}(\Omega)\right) \\
& u(0) \leftarrow u_{0 m}:=\sum_{v=1}^{m}\left(u_{0}, w_{v}\right) w_{v} \rightarrow u_{0}
\end{aligned}
$$

Se concluye que $u(0)=u_{0}$

Etapa3. Unicidad de la solución generalizada

$\forall \mathrm{v} \in \mathrm{H}_{0}^{1}(\Omega)$ en el sentido de la $\mathrm{D}^{\prime}(0, \mathrm{~T})$, se tiene 


\section{La identidad de Parseval}

Si $f \in \mathrm{L}^{2},\|\hat{\mathrm{f}}\|_{\mathrm{L}^{2}}=\|\mathrm{f}\|_{\mathrm{L}^{2}}$

, donde $\hat{\mathrm{f}}(\mathrm{x})=\int \mathrm{f}(\mathrm{t}) \mathrm{e}^{-2 \pi \mathrm{ixt}} \mathrm{dt}$

(Ortiz, 1988)

\section{MATERIAL Y MÉTODOS}

$\left\{\begin{array}{c}\frac{\mathrm{d}}{\mathrm{dt}}(\mathrm{u}(\mathrm{t}), \mathrm{v})+\mathrm{a}(\mathrm{u}(\mathrm{t}), \mathrm{v})=(\mathrm{f}(\mathrm{t}), \mathrm{v}) \\ \mathrm{u}(0)=u_{0}\end{array}\right.$

Sean $\mathrm{u}_{1} \mathrm{y} \mathrm{u}_{2}$ dos soluciones del problema

(12) tal que

$i=1,2,\left\{\begin{array}{c}\frac{\mathrm{d}}{\mathrm{dt}}\left(u_{i}(\mathrm{t}), \mathrm{v}\right)+\mathrm{a}\left(u_{i}(\mathrm{t}), \mathrm{v}\right)=(\mathrm{f}(\mathrm{t}), \mathrm{v}) \\ u_{i}(0)=u_{0}\end{array}\right.$

(13)

Restando las dos ecuaciones en (13) se obtiene

$\left\{\begin{array}{c}\frac{\mathrm{d}}{\mathrm{dt}}\left(u_{1}(\mathrm{t})-\mathrm{u}_{2}(\mathrm{t}), \mathrm{v}\right)+\mathrm{a}\left(\mathrm{u}_{1}(\mathrm{t})-\mathrm{u}_{2}(\mathrm{t}), \mathrm{v}\right)=(\mathrm{f}(\mathrm{t}), \mathrm{v}) \\ \left(\mathrm{u}_{1}-\mathrm{u}_{2}\right)(0)=\mathrm{u}_{1}(0)-\mathrm{u}_{2}(0)=\mathrm{u}_{0}-\mathrm{u}_{0}=0\end{array}\right.$

En particular sea $\mathrm{w}=\mathrm{u}_{1}-\mathrm{u}_{2} \in \mathrm{H}_{0}^{1}(\Omega)$ y reemplazando en (14) se tiene

$\left\{\begin{array}{c}\frac{\mathrm{d}}{\mathrm{dt}}(\mathrm{w}(\mathrm{t}), \mathrm{w}(\mathrm{t}))+\mathrm{a}(\mathrm{w}(\mathrm{t}), \mathrm{w}(\mathrm{t}))=0 \\ w(0)=u_{0}\end{array}\right.$

(15)

Por propiedad de producto interno en

(15), se cumple

$$
\begin{aligned}
&\left\{\frac{\mathrm{d}}{\mathrm{dt}}|\mathrm{w}(\mathrm{t})|_{\mathrm{L}^{2}(\Omega)}^{2}+\mathrm{a}(\mathrm{w}(\mathrm{t}), \mathrm{w}(\mathrm{t}))=0\right. \\
& \mathrm{w}(0)=\mathrm{u}_{0} \\
& \frac{\mathrm{d}}{\mathrm{dt}}|w(t)|_{\mathrm{L}^{2}(\Omega)}^{2} \\
& \leq \frac{\mathrm{d}}{\mathrm{dt}}|w(t)|_{\mathrm{L}^{2}(\Omega)}^{2} \\
&+\alpha\|w(t)\|_{\mathrm{H}_{0}^{1}(\Omega)}^{2} \leq \\
& \leq \frac{\mathrm{d}}{\mathrm{dt}}|w(t)|_{\mathrm{L}^{2}(\Omega)}^{2} \\
&+\mathrm{a}(\mathrm{w}(\mathrm{t}), \mathrm{w}(\mathrm{t}))=0
\end{aligned}
$$

Pero, de (16) se obtiene

$$
\left\{\begin{array}{c}
\frac{d}{d t}|w(t)|_{L^{2}(\Omega)}^{2} \leq 0 \\
w(0)=u_{0}
\end{array}\right.
$$

(17)

Sean $\varphi(t)=|w(t)|_{L^{2}(\Omega)}^{2}$,

$c_{1}=c_{2}=0$. Aplicando el lema de

Gronwall se obtiene

$\varphi(\mathrm{t}) \leq 0$, entonces

$|\mathrm{w}(\mathrm{t})|_{\mathrm{L}^{2}(\Omega)}^{2}=0 \Rightarrow \mathrm{w}(\mathrm{t})=0$.

Por lo tanto $\mathrm{u}_{1}(\mathrm{t})=\mathrm{u}_{2}(\mathrm{t})$

\section{CONCLUSIONES}

Al considerar un problema de valor inicial y de frontera en el caso que puedan poseer soluciones no diferenciables en el sentido clásico e incluso discontinua, se estableció la existencia y unicidad de una solución débil o generalizada para una ecuación diferencial parabólica que modela la distribución de temperatura sobre la frontera de un dominio cilíndrico sometida a una fuerza externa.

\section{REFERENCIAS BIBLIOGRÁFICAS}

Adams, R. (1975). Sobolev Space. Nueva York: Academic Press.

Boyer, C. (1986). Historia de la matemática. Madrid: Alianza Universidad Textos.

Brézis, H. (1984). Análisis Funcional. Madrid: Alianza Editorial.Medeiros, L.A. (1990). Ecuaciones diferenciales de evolución. Brasil: IMUFRJ.

Ian, S. (2012). Historia de la matemática en los últimos 10000 años .Disponible en http/:www.librosmaravillosos.com.

Ortiz, A. (1988). Aspectos básicos en ecuaciones en derivadas parciales. Trujillo: Departamento de Matemática de la Universidad Nacional de Trujillo: 124142.Print.

Vera, E. (1984). Una introducción a las ecuaciones diferenciales parciales. Lima: Sociedad Matemática Peruana Segundo Coloquio.

VP 